\title{
Sums of Squares: A Real Projective Story
}

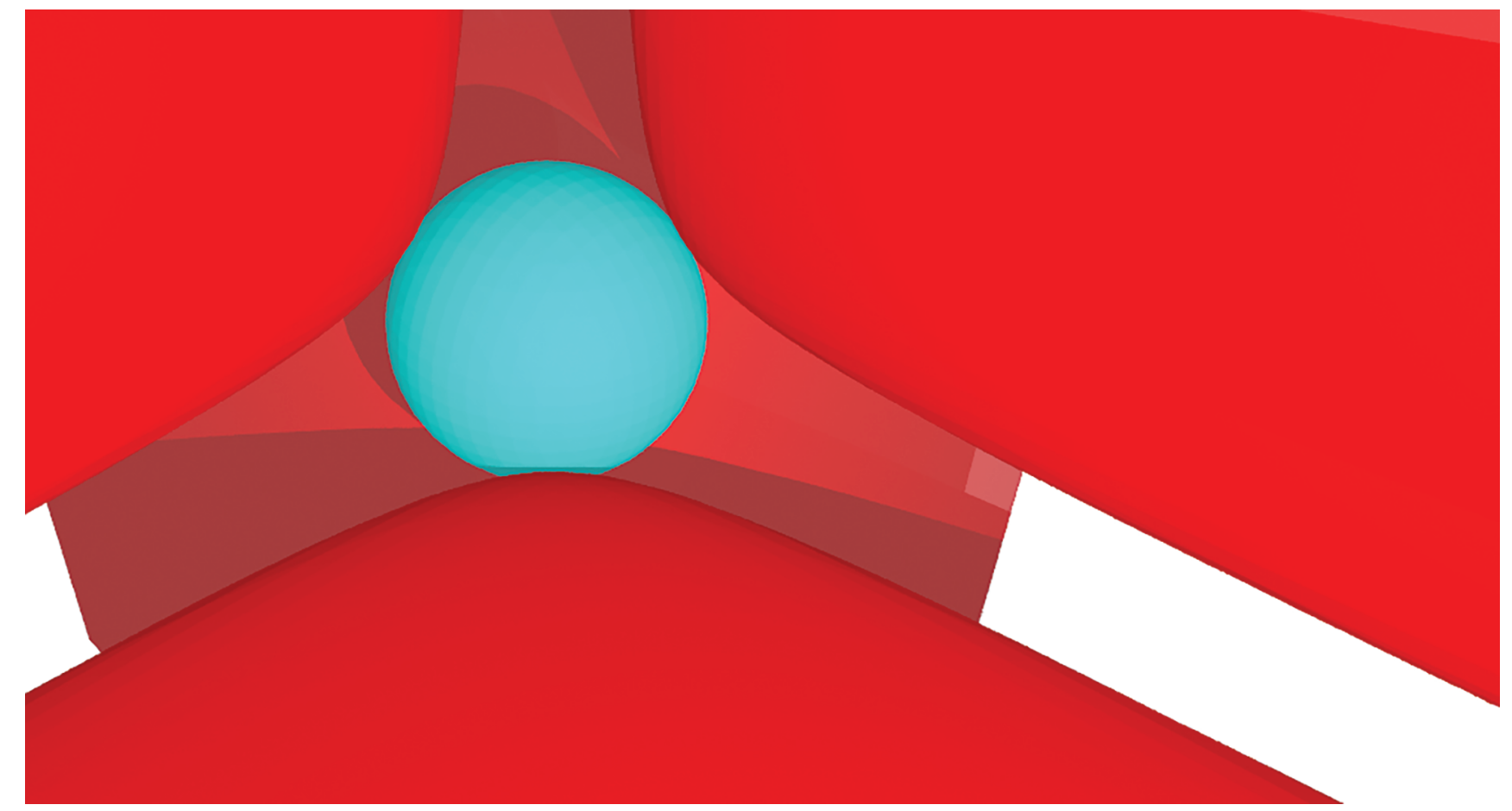

\section{Grigoriy Blekherman, Rainer Sinn, Gregory G. Smith, and Mauricio Velasco}

How do we find the minimum of the function $2\left(x^{2}-y z\right)$ on the unit sphere? An algebraic way to see that the minimum equals -1 involves the decomposition

$$
2\left(x^{2}-y z\right)+1-\left(1-x^{2}-y^{2}-z^{2}\right)=3 x^{2}+(y-z)^{2} .
$$

The unit sphere in $\mathbb{R}^{3}$ is the zero-locus of the polynomial $1-x^{2}-y^{2}-z^{2}$. This decomposition establishes that the

Grigoriy Blekherman is a professor of mathematics at the Georgia Institute of Technology. His email address is greg@math. gatech. edu.

Rainer Sinn is a professor of mathematics at the University of Leipzig. His email address is rainer.sinn@uni-1eipzig.de.

Gregory G. Smith is a professor of mathematics at Queen's University. His email address is ggsmi th@mast. queensu.ca.

Mauricio Velasco is a professor of mathematics at the Universidad de los Andes. His email address is mve1asco@uniandes.edu.co.

Communicated by Notices Associate Editor Steven Sam.

For permission to reprint this article, please contact:

reprint-permission@ams.org.

DOI: https://doi.org/10.1090/noti2280 function $2\left(x^{2}-y z\right)+1$ is a sum of squares modulo the defining equation of the unit sphere, so this function is nonnegative on the sphere. Thus, we have a certificate that the minimum of $2\left(x^{2}-y z\right)$ on the unit sphere is at least -1 . This lower bound is optimal because the sum of squares $3 x^{2}+(y-z)^{2}$ vanishes at the points $\pm \frac{1}{\sqrt{2}}(0,1,1)$ on the unit sphere. Figure 1 illustrates that the unit sphere is tangent to the zero-locus of $2\left(x^{2}-y z\right)+1$ at these two points.

This example indicates that nonnegativity is intimately related to polynomial optimization and sum-of-squares representations give rise to lower bounds on minima. Does this approach lead to sharp bounds for all quadratic polynomials? Does it apply to higher-degree polynomials on the unit sphere? What happens when we replace the unit sphere with another algebraic variety? This article examines these basic questions. In the process, we also uncover fascinating connections between real and complex algebraic geometry. Remarkably, convex geometry provides the bridge between these two worlds. 


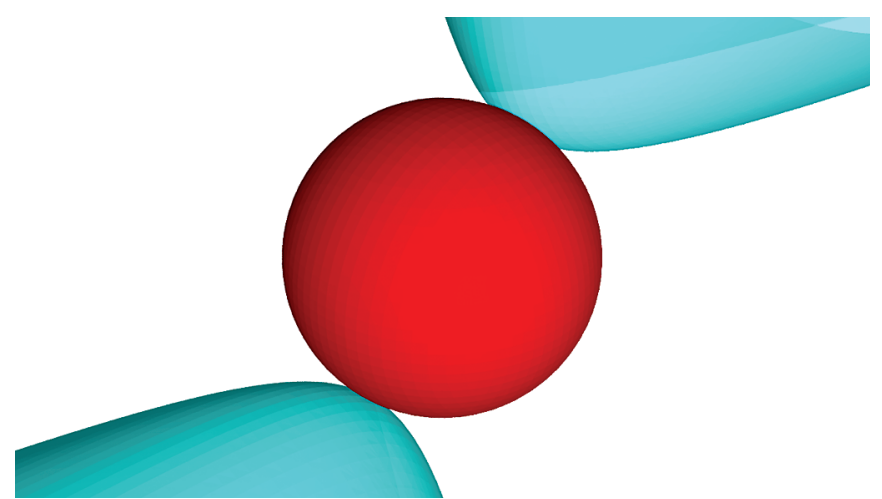

Figure 1. The unit sphere in red and the zero-locus of $2\left(x^{2}-y z\right)+1$ in teal.

As a second motivating example, let $X$ be the cubic surface defined as the real zero-locus of the polynomial $q:=x y z-w^{3}$ and consider $f:=x^{2}+y^{2}+z^{2}-3 w^{2}$. This quadratic polynomial is not a sum of squares modulo the defining ideal of $X$ because no nonzero polynomial of degree less than 3 vanishes on the algebraic variety $X$ and $f$ is not globally nonnegative. Nevertheless, we claim that $f$ is nonnegative on the algebraic variety $X$. We prove this in Section 1 by recognizing $f$ as the Motzkin polynomial in disguise. More directly, we certify the nonnegativity of $f$ on $X$ with the sum-of-squares decomposition

$$
g f-12 w q=h_{1}^{2}+h_{2}^{2}+\cdots+h_{6}^{2},
$$

where $g:=x^{2}+y^{2}+z^{2}+(\sqrt{3} w)^{2}$ is a sum of squares, and the squares on the right are given by $h_{1}:=x^{2}-w^{2}$, $h_{2}:=\sqrt{2}(x y-z w), h_{3}:=\sqrt{2}(x z-y w), h_{4}:=y^{2}-w^{2}$, $h_{5}:=\sqrt{2}(y z-x w)$, and $h_{6}:=z^{2}-w^{2}$. The important new ingredient is the multiplier $g$. As $g$ is nonnegative and not divisible by the irreducible polynomial $q$, this decomposition confirms that $f \geqslant 0$ whenever $q=0$. Equivalently, the function $f$ is the sum $\sum_{i} h_{i}^{2} / g$ of nonnegative rational functions on the variety $X$. Restricting to $w=1$, Figure 2 depicts the variety $X$ as tangent to the sphere defined by $x^{2}+y^{2}+z^{2}=3$ at the points $(1,1,1),(1,-1,-1),(-1,1,-1)$, and $(-1,-1,1)$. Thus, the restriction of the function $f$ to $X \cap\{w \neq 0\}$ has a minimum value equal to zero.

To understand the relationship between nonnegativity and sums of squares, it suffices to consider homogeneous polynomials. Indeed, homogenizing any sum-of-squares decomposition by introducing a new variable produces a homogeneous sum-of-squares decomposition. It follows that a polynomial is a sum of squares modulo the ideal of an affine subvariety $X \subseteq \mathbb{A}^{n}$ if and only if an appropriate homogenization (having sufficiently high degree) is a sum of squares modulo the homogeneous ideal of the projective completion $\bar{X} \subseteq \mathbb{P}^{n}$.

Working with projective varieties has conceptual and technical advantages, so we concentrate on nonnegative

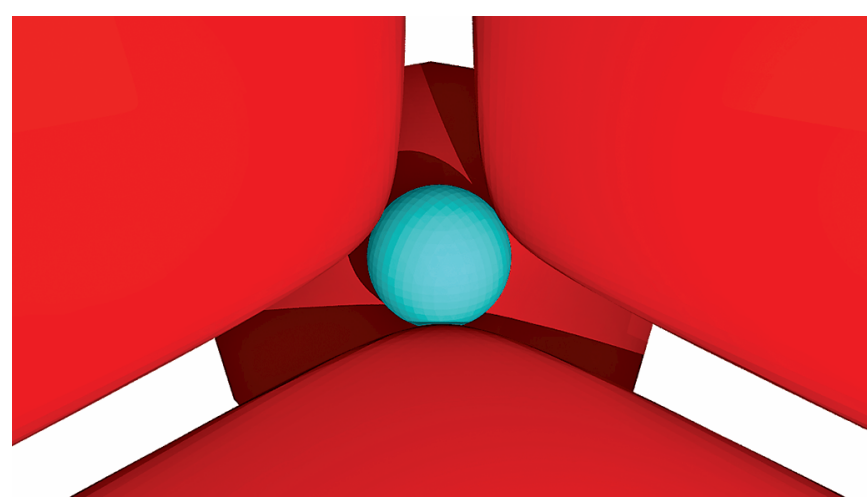

Figure 2. The real zero-loci of $x y z-1$ in red and $x^{2}+y^{2}+z^{2}-3$ in teal.

functions and sums of squares on real subvarieties of projective space. We examine three problems:

(1) Identify all of the real projective varieties on which every nonnegative quadratic function is a polynomial sum of squares.

(2) Knowing that a nonnegative function is a polynomial sum of squares on a projective variety, control the number of squares needed in such a decomposition.

(3) Bound the degree of a sum-of-squares multiplier such that its product with a nonnegative function decomposes into a polynomial sum of squares.

Each of the subsequent sections is devoted to one of these problems. Sections begin with some historial context before describing more recent results.

Sums of squares of polynomials are indispensable in the theory of nonnegative polynomials. Beyond forming a self-evident subset of nonnegative polynomials, there are practical algorithms for deciding whether a given polynomial is a sum of squares. The existence of a polynomial sum-of-squares decomposition is equivalent to the feasibility of a semidefinite programming problem: a convex optimization problem with a linear objective function on the intersection of the cone of positive semidefinite matrices with an affine linear space. There are robust and efficient software packages, typically based on interior point methods, for solving these semidefinite programming problems. More theoretically, polynomial sums of squares generate an infinite hierarchy of approximations for the set of nonnegative polynomials. The resulting sum-of-squares relaxations (also known as Lasserre relaxations) play a prominent role in engineering applications and computer science; see Chapter 7 in [4].

\section{Geometry of Sums of Squares}

When is every nonnegative polynomial a sum of squares? For which degrees and in how many variables is there an equivalence? David Hilbert gave a eulogy [17] in 1910 for Hermann Minkowski (1864-1909) to the Royal Society of Sciences in Göttingen. Hilbert explained that the history 
of this question began in 1885 with Minkowski's thesis defense. Hilbert participated in this defense as an examiner. Two years prior to the defense, Minkowski made a name for himself by solving a problem posed by Eisenstein in a competition for the Academy of Sciences (Paris) about the number of representations of a positive integer as a sum of five squares. His doctoral thesis was a continuation of this prize-winning work. In his defense, Minkowski argued that there are polynomials with real coefficients that are nonnegative functions on Euclidean space but cannot be written as sums of squares of polynomials.

Minkowski never published these insights, but he did inspire Hilbert's seminal work in the area. Hilbert [15] demonstrated, in 1888, that Minkowski's claim is correct by completely characterizing when every nonnegative polynomial is a polynomial sum of squares.

Theorem 1.1 (Hilbert). Every nonnegative homogeneous polynomial on $\mathbb{R}^{n}$ having degree $2 d$ is a sum of squares of polynomials if and only if

- $2 d=2$,

- $n=2$, or

- $n=3$ and $2 d=4$.

(quadratic forms)

(binary forms)

(ternary quartics)

In a second paper, Hilbert [16] also characterized nonnegative polynomials in two variables (or equivalently homogeneous polynomials in three variables) as sums of squares of rational functions. These results undoubtably prompted Hilbert to formulate the 17th problem on his celebrated list of 23 problems.

For the first two cases in Theorem 1.1, establishing that all nonnegative polynomials are sums of squares of polynomials is relatively straightforward. The third case is noticeably more challenging. Arguably, the hardest part is proving that there exist nonnegative polynomials that are not polynomial sums of squares in all of the remaining cases. Hilbert accomplished this task with an impressive nonconstructive argument.

Curiously, Theodore Motzkin published in 1967, nearly 80 years after Hilbert's paper, the first explicit example of a nonnegative polynomial that cannot be expressed as a polynomial sum of squares. The Motzkin polynomial is the ternary sextic $x_{0}^{6}+x_{1}^{4} x_{2}^{2}+x_{1}^{2} x_{2}^{4}-3 x_{0}^{2} x_{1}^{2} x_{2}^{2}$. By taking suitable means of $x_{0}^{2}, x_{1}^{2}$, and $x_{2}^{2}$, the nonnegativity of this homogeneous polynomial on $\mathbb{R}^{3}$ follows immediately from the arithmetic-geometric mean inequality.

Real projective varieties. As alluded in the preceding section, it is advantageous to work with homogeneous polynomials and real projective varieties. Within this broader framework, we not only generalize Theorem 1.1 but discover a geometric characterization of the equality between nonnegative polynomials and sums of squares.

We consider the $n$-dimensional projective space $\mathbb{P}^{n}$ to be the set of lines in $\mathbb{C}^{n+1}$ passing through the origin. The point $p:=\left[p_{0}: p_{1}: \cdots: p_{n}\right] \in \mathbb{P}^{n}$ is the equivalence class

$$
\left\{\left(\lambda p_{0}, \lambda p_{1}, \ldots, \lambda p_{n}\right) \in \mathbb{C}^{n+1} \backslash\{\mathbf{0}\} \mid \text { for some } 0 \neq \lambda \in \mathbb{C}\right\} .
$$

The point $p$ is real if it has a representative $\left[p_{0}: p_{1}: \cdots: p_{n}\right]$ such that $p_{i} \in \mathbb{R}$ for all $0 \leqslant i \leqslant n$.

A real subvariety $X \subseteq \mathbb{P}^{n}$ is the set of common zeroes of some homogeneous polynomials with real coefficients in the variables $x_{0}, x_{1}, \ldots, x_{n}$. Whenever a homogeneous polynomial $f$ vanishes at $p:=\left(p_{0}, p_{1}, \ldots, p_{n}\right) \in \mathbb{C}^{n+1}$, it also vanishes at all scalar multiples because homogeneity implies that $f(\lambda p)=\lambda^{\operatorname{deg}(f)} f(p)$ for all nonzero scalars $\lambda$. In other words, the homogeneous polynomial $f$ vanishes at the point $\left[p_{0}: p_{1}: \cdots: p_{n}\right] \in \mathbb{P}^{n}$ and the set of common zeroes of a collection of homogeneous polynomials is a subset of $\mathbb{P}^{n}$. For example, the twisted cubic curve

$$
C:=\left\{\left[t_{0}^{3}: t_{0}^{2} t_{1}: t_{0} t_{1}^{2}: t_{1}^{3}\right] \in \mathbb{P}^{3} \mid\left[t_{0}: t_{1}\right] \in \mathbb{P}^{1}\right\}
$$

is the set of common zeroes of the quadratic polynomials $x_{0} x_{2}-x_{1}^{2}, x_{0} x_{3}-x_{1} x_{2}$, and $x_{1} x_{3}-x_{2}^{2}$.

Throughout, we assume that the subvariety $X \subseteq \mathbb{P}^{n}$ is nondegenerate and totally real. The first assumption means that the subvariety $X$ does not lie in a hyperplane. This mild hypothesis guarantees that the ambient projective space $\mathbb{P}^{n}$ is not unnecessarily large. The second assumption is more significant-it ensures that $X$ has enough real points. By definition, the subvariety $X$ is totally real if the set of real points in $X$, regarded as a subset of the complex points in $X$, is dense in the Zariski topology. Equivalently, every irreducible component of the algebraic variety $X$ has a nonsingular real point. The twisted cubic curve in $\mathbb{P}^{3}$ is totally real whereas the zero set of the polynomial $x_{0}^{2}+x_{1}^{2}+\cdots+x_{n}^{2}$ is not because it does not contain a real point in $\mathbb{P}^{n}$.

In this geometric context, polynomials are replaced by elements in another ring. The homogeneous coordinate ring $R$ of the subvariety $X \subseteq \mathbb{P}^{n}$ is the quotient of the polynomial ring $\mathbb{R}\left[x_{0}, x_{1}, \ldots, x_{n}\right]$ by the ideal generated by all homogeneous polynomials that vanish on $X$. For any nonnegative integer $j$, let $R_{j}$ denote the real vector space spanned by the homogeneous elements in $R$ of degree $j$. Generalizing the concept of a polynomial sum of squares is not difficult. A homogeneous element $f \in R_{2 j}$ is a sum of squares on $X$ if there exist homogeneous elements $h_{1}, h_{2}, \ldots, h_{r} \in R_{j}$ such that $f=h_{1}^{2}+h_{2}^{2}+\cdots+h_{r}^{2}$.

In comparison, defining nonnegativity requires more care. A homogeneous element $f \in R_{2 j}$ of even degree is nonnegative on $X$ if its evaluation at each real point in $X$ is greater than or equal to 0 . Since elements in the ring $R$ and points in the space $\mathbb{P}^{n}$ may both be thought of as equivalence classes, the evaluation process involves choosing a polynomial in $\mathbb{R}\left[x_{0}, x_{1}, \ldots, x_{n}\right]$ to represent the ring element and a point in $\mathbb{R}^{n+1}$ to represent the real point in $X \subset \mathbb{P}^{n}$. Although a homogeneous element $f$ in $R_{2 j}$ does not determine a function from $X$ to $\mathbb{R}$, evaluating 
its polynomial representative at a representative point in $\mathbb{R}^{n+1}$ does have a well-defined sign because the degree of polynomial is even. We recover our original notion of nonnegativity for polynomials when $X=\mathbb{P}^{n}$.

Example 1.2. Since we have

$$
x_{0}^{2}-x_{0} x_{3}+x_{1} x_{2}+x_{2} x_{3}=x_{0}^{2}+x_{2} x_{3}-\left(x_{0} x_{3}-x_{1} x_{3}\right),
$$

both $x_{0}^{2}-x_{0} x_{3}+x_{1} x_{2}+x_{2} x_{3}$ and $x_{0}^{2}+x_{2} x_{3}$ represent the same element in the homogeneous coordinate ring of the twisted cubic curve $C$. Choosing $(1,0,0,0) \in \mathbb{R}^{4}$ or $(-2,0,0,0) \in \mathbb{R}^{4}$ as representatives for the real point $[1: 0: 0: 0] \in C$, either of the evaluations

$$
(1)^{2}-(1)(0)+(0)(0)+(0)(0)=1>0
$$

or $(-2)^{2}+(0)(0)=4>0$ show that this element in the homogeneous coordinate ring is positive at this point. $\diamond$

Sums of squares on real projective varieties. The real vector space $R_{2}$ of quadrics on the real subvariety $X \subseteq \mathbb{P}^{n}$ contains two fundamental subsets:

- The set $P_{X} \subseteq R_{2}$ consists of those elements whose evaluations at every real point in $X$ is nonnegative;

$P_{X}:=\left\{f \in R_{2} \mid f(x) \geqslant 0\right.$ for all real points $\left.x \in X\right\}$.

- The set $\Sigma_{X} \subseteq R_{2}$ consists of sums of squares;

$$
\Sigma_{X}:=\left\{\sum_{i} h_{i}^{2} \mid h_{i} \in R_{1} \text { for all } i\right\} .
$$

As the square of any real number is nonnegative, we have the inclusion $\Sigma_{X} \subseteq P_{X}$. Following Hilbert [15], we ask the following question.

Question 1.3. For which subvarieties $X \subseteq \mathbb{P}^{n}$ is every nonnegative quadratic element in $R$ a sum of squares? Equivalently, when does the equality $\Sigma_{X}=P_{X}$ hold?

At first glance, focusing on just the quadratic elements seems to be a limited generalization of Hilbert's work. However, the elbow room gained by considering all real projective subvarieties alleviates this concern. Suppose that we are interested in the homogeneous elements of degree $2 j$ on a subvariety $X \subseteq \mathbb{P}^{n}$. Set $m:=\left(\begin{array}{c}n+j \\ j\end{array}\right)-1$ and let $\nu_{j}: \mathbb{P}^{n} \rightarrow \mathbb{P}^{m}$ be the $j$ th Veronese map that sends the point $\left[p_{0}: p_{1}: \cdots: p_{n}\right] \in \mathbb{P}^{n}$ to the point in $\mathbb{P}^{m}$ whose coordinates are all possible monomials of degree $j$ evaluated at $\left(p_{0}, p_{1}, \ldots, p_{n}\right)$. By re-embedding the subvariety $X \subseteq \mathbb{P}^{n}$, it is enough to understand the quadratic elements on the image $\nu_{j}(X) \subset \mathbb{P}^{m}$.

Example 1.4. On the twisted cubic curve $C$, the set $P_{C}$ may be identified with the homogeneous polynomials in $\mathbb{R}\left[t_{0}, t_{1}\right]$ having degree 6 that are nonnegative on $\mathbb{P}^{1}$. Likewise, the set $\Sigma_{X}$ may be identified with sums of squares of homogeneous polynomials in $\mathbb{R}\left[t_{0}, t_{1}\right]$ having degree 3 . By Theorem 1.1, these sets coincide, so $P_{C}=\Sigma_{C}$.
Example 1.5. A variant of the Veronese map shows that the Motzkin polynomial $x_{0}^{6}+x_{1}^{4} x_{2}^{2}+x_{1}^{2} x_{2}^{4}-3 x_{0}^{2} x_{1}^{2} x_{2}^{2}$ is not a sum of squares of cubic polynomials. If it were, then an easy (if somewhat tedious) case study shows that the cubic polynomials would only involve the monomials $x_{0}^{3}, x_{1}^{2} x_{2}, x_{1} x_{2}^{2}, x_{0} x_{1} x_{2}$. Alternatively, one may use Newton polytopes (the convex hull of the exponent vectors) to identify these monomials. The Newton polytope of a sum of squares contains the Newton polytopes of every summand, and the Newton polytope of a product is the Minkowski sum of the Newton polytopes of the factors. So consider the map $\mathbb{P}^{2} \rightarrow \mathbb{P}^{3}$ defined by

$$
\left[x_{0}: x_{1}: x_{2}\right] \mapsto\left[x_{0}^{3}: x_{1}^{2} x_{2}: x_{1} x_{2}^{2}: x_{0} x_{1} x_{2}\right] .
$$

The image of this map is the zero-locus of the polynomial $q:=x y z-w^{3}$ where the homogeneous coordinate ring of $\mathbb{P}^{3}$ is $\mathbb{R}[x, y, z, w]$. Hence, the Motzkin polynomial is the restriction of the function $f:=x^{2}+y^{2}+z^{2}-3 w^{2}$ to the image variety. As explained in the first section, this quadratic polynomial is not a sum of squares modulo the defining ideal of the surface because no nonzero polynomial of degree less than 3 vanishes on the image variety and $f$ is not nonnegative on $\mathbb{P}^{3}$. Therefore, the Motzkin polynomial cannot be a polynomial sum of squares.

The surprising answer to Question 1.3, restated in the next theorem, first appeared as Theorem 1.1 in [8]. To formulate this result, recall that a variety is irreducible if it is not the union of two proper subvarieties. From the algebraic point of view, a variety is irreducible if and only if the ideal of polynomials vanishing on it is prime.

Theorem 1.6 (Blekherman, Smith, and Velasco). Let $X$ be an irreducible nondegenerate totally-real subvariety in $\mathbb{P}^{n}$. Every nonnegative quadratic function on $X$ is a polynomial sum of squares, modulo the defining ideal of $X$, if and only if we have $\operatorname{deg}(X)=\operatorname{codim}(X)+1$.

This theorem reveals a remarkable connection between a semialgebraic property (any nonnegative polynomial being a polynomial sum of squares) and the fundamental geometric invariants of the complex variety. For any subvariety $X \subseteq \mathbb{P}^{n}$, the codimension is a simple numerical measure of its relative size; $\operatorname{codim}(X):=n-\operatorname{dim}(X)$. The degree is a second numerical invariant depending on the embedding of the variety $X$ in $\mathbb{P}^{n}$. Geometrically, the degree, denoted by $\operatorname{deg}(X)$, is the number of points in the intersection of the variety $X$ and a general linear subspace of dimension $\operatorname{codim}(X)$. For instance, if we intersect the twisted cubic $C=\left\{\left[t_{0}^{3}: t_{0}^{2} t_{1}: t_{0} t_{1}^{2}: t_{1}^{3}\right]\right\} \subset \mathbb{P}^{3}$ with the generic hyperplane given by the linear equation $a x_{0}+b x_{1}+c x_{2}+d x_{3}=0$, then the intersection points correspond to the three (typically distinct) roots of the binary cubic $a t_{0}^{3}+b t_{0}^{2} t_{1}+c t_{0} t_{1}^{2}+d t_{1}^{3}, \operatorname{sodeg}(C)=3$. As the 
codimension of the curve $C$ in $\mathbb{P}^{3}$ is 2 , Theorem 1.6 again implies that $P_{C}=\Sigma_{C}$; compare with Example 1.4.

The irreducible nondegenerate subvarieties $X \subset \mathbb{P}^{n}$ satisfying $\operatorname{deg}(X)=\operatorname{codim}(X)+1$ are called varieties of minimal degree. As the terminology suggests, these subvarieties do have the smallest possible degree. The complete classification of varieties of minimal degree is one of the outstanding achievements of the classical Italian school of algebraic geometry. Pasquale del Pezzo (1886) classified the surfaces of minimal degree and Eugenio Bertini (1907) extended it to varieties of any dimension; see [13] for an account. The next subsection summarizes this result and highlights links with sums of squares. The remainder of this subsection is devoted to two ideas: the impact of the minimal degree condition and the pivotal role played by convex geometry, which has so far been hidden.

To get a feel for the degree condition, we temporarily assume that the subvariety $X$ is a finite set of real points in $\mathbb{P}^{n}$. Although this variety is reducible whenever there is more than one point, this zero-dimensional case nevertheless develops some valuable intuition. If $X$ is a variety of minimal degree, then it is a set of $n+1$ real points which span the ambient space $\mathbb{P}^{n}$. Hence, we may choose coordinates on $\mathbb{P}^{n}$ such that $X=\left\{\left[e_{0}\right],\left[e_{1}\right], \ldots,\left[e_{n}\right]\right\}$, where $e_{1}, e_{2}, \ldots, e_{n}$ are the standard basis vectors for $\mathbb{R}^{n+1}$.

Why can we express every nonnegative quadratic form as a polynomial sum of squares on this zero-dimensional variety? The answer essentially comes from interpolation. The coordinate function $x_{i}$ vanishes at all points in $X$ except $\left[e_{i}\right]$ for all $0 \leqslant i \leqslant n$. It follows that, for any nonnegative quadratic form $q$ on $X$, the equality

$$
q=\sum_{i=0}^{n}\left(\sqrt{q\left(e_{i}\right)} x_{i}\right)^{2}
$$

holds in the homogeneous coordinate ring of $X$, proving that $q$ is a polynomial sum of squares. If the subvariety $X$ has more than $n+1$ real points (which implies that $\operatorname{deg}(X)>\operatorname{codim}(X)+1)$, then the interpolation is no longer possible, because there exists a nontrivial linear relation between the values of the linear forms at the points on $X$. These linear relations impose constraints on the possible values of quadratic sums of squares on $X$ allowing us to separate $P_{X}$ and $\Sigma_{X}$.

For the surface $X=\nu_{3}\left(\mathbb{P}^{2}\right) \subset \mathbb{P}^{9}$, Hilbert [15] already used these linear relations to prove that the cone $\Sigma_{X}$ of sums of squares is properly contained in the cone $P_{X}$ of nonnegative polynomials. A hyperplane section of the subvariety $X$ corresponds to a cubic curve in the plane. Cutting $X$ with two hyperplanes, one obtains the intersection of two cubic curves in the plane. By Bézout's Theorem, two general cubics intersect in $9=3 \cdot 3$ points, so $\operatorname{deg}(X)=9=\operatorname{codim}(X)+2$. By choosing the two cubics appropriately, we can arrange for the intersection points to all be real. These nine points of intersection lie in $\mathbb{P}^{7}$ and are therefore linearly dependent. Moreover, the values of cubic forms on these points are also linearly related. In classical algebraic geometry, this is known as the CayleyBacharach theorem and is stated as: any cubic passing through any eight points of the intersection must also pass through the ninth point. Exploiting this linear relation, Hilbert showed that sum-of-square cone $\Sigma_{X}$ is properly contained in the nonnegativity cone $P_{X}$. Robinson later used Hilbert's technique to construct an explicit nonnegative ternary form that is not a sum of squares; see [23].

What serves as the bridge between complex algebraic geometry and semialgebraic nonnegativity results? For the work under discussion, the unifying answer is convex geometry. Both $P_{X}$ and $\Sigma_{X}$ are more than just subsets of the vector space $R_{2}$ of quadrics on the subvariety $X \subseteq \mathbb{P}^{n}$. They are closed convex cones. As convex cones, these subsets are closed under taking linear combinations with nonnegative coefficients. Being closed means that these subsets are closed sets in the natural Euclidean topology on the real vector space $R_{2}$.

Duality is an intrinsic feature of convex geometry. Every closed convex cone is equal to the intersection of the closed half-spaces that contain it. The linear inequalities defining these closed half-spaces form a convex cone in the dual vector space, unimaginatively called the dual cone. The most accessible convex cones are polyhedral, defined by finitely many linear inequalities. Neither $P_{X}$ nor $\Sigma_{X}$ is a polyhedral cone. Their dual cones can be quite complicated. Fortuitously, the dual cone $\Sigma_{X}^{*}$ belongs to the next best class of convex cones.

For this part of our story, the cone of positive semidefinite matrices plays a distinguished role. The positive semidefinite quadratic forms constitute the closed convex cone $\mathbb{S}_{+}^{n+1}$ inside the real vector space $\mathbb{R}\left[x_{0}, x_{1}, \ldots, x_{n}\right]_{2}$ of quadratic polynomials. Moreover, the sum-of-squares cone $\Sigma_{X}$ is the image of the cone $\mathbb{S}_{+}^{n+1}$ under the canonical linear projection from $\mathbb{R}\left[x_{0}, x_{1}, \ldots, x_{n}\right]_{2}$ to $R_{2}$. It follows that the dual cone $\Sigma_{X}^{*}$ is spectrahedral-this cone can be represented as a linear matrix inequality or equivalently as the intersection of $\mathbb{S}_{+}^{n+1}$ with a linear affine subspace. Understanding the minimal generators of the spectrahedral cone $\Sigma_{X}^{*}$ underpins our advancements.

Convex duality also produces fruitful connections between real algebraic geometry and real analysis. The dual cone $P_{X}^{*}$ consists of the linear functionals that are nonnegative on $P_{X}$. Given a linear functional that evaluates nonnegatively on nonnegative functions, one hopes that it may be represented as integration with respect to a measure supported on $X$. This forecast is often correct. For example, the equality between nonnegative polynomials and sums of squares in the univariate leads to a solution of the Hamburger, Hausdorff, and Stiltjes moment problems; see Chapters 3 and 9 in [25]. 
The classification. Varieties of minimal degree come in three flavors. The most palatable are quadratic hypersurfaces. The real quadratic forms satisfying the hypothesis of Theorem 1.6 are necessarily indefinite. Specializing to this case, the theorem asserts that, when a quadratic form is nonnegative on the set of real points at which an indefinite quadratic form vanishes, there exists a linear combination of the two forms that is positive semidefinite. This statement is equivalent to the well-known $S$-Lemma (or $S$-procedure) in the optimization community. When $X=\mathbb{P}^{n}$, this covers the quadratic forms in Theorem 1.1.

The second flavor is an infinite family of projective varieties called rational normal scrolls. Every member in this family is a smooth toric variety, but there are infinitely many in each dimension. The one-dimensional family members are the rational normal curves arising as the Veronese embeddings of $\mathbb{P}^{1}$. This family corresponds to the binary forms in Theorem 1.1.

The third flavor consists of just one variety, namely the Veronese surface in $\mathbb{P}^{5}$. This projective variety is defined by $\left\{\left[x^{2}: x y: x z: y^{2}: y z: z^{2}\right] \in \mathbb{P}^{5} \mid[x: y: z] \in \mathbb{P}^{2}\right\}$. This exceptional variety corresponds to the sporadic case of the ternary quartics in Theorem 1.1.

The structure of the rational normal scrolls warrants a closer look. The surfaces in this family are toric varieties corresponding to lattice polygons of the form $\operatorname{conv}\{(0,0),(0,1),(d, 0),(e, 1)\}$; see Figure 3. This polygon

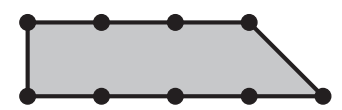

Figure 3. The polygon of the rational normal scroll when $d=4$ and $e=3$.

defines a map from the torus $\left(\mathbb{C}^{*}\right)^{2}$ to $\mathbb{P}^{d+e+1}$ given by $(s, t) \mapsto\left[1: t: t^{2}: \cdots: t^{d}: s: s t: \cdots: s t^{e}\right]$, where monomials have exponent vectors equal to the lattice points of the polygon. The closure of the image is the rational normal scroll corresponding to the polygon. It is a projective embedding of the $(d-e)$ th Hirzebruch surface. There are two rational normal curves, one of degree $d($ for $s=0)$ and one of degree $e$ (for $s=\infty$ ), contained in this surface. The scroll is swept out by the lines joining the two points on the rational normal curves for the same values of $t$. Assuming that $d \geqslant e$, one may homogenize and write as the map $\mathbb{P}^{1} \times \mathbb{P}^{1} \rightarrow \mathbb{P}^{d+e+1}$ sending the pair $\left(\left[s_{0}: s_{1}\right],\left[t_{0}: t_{1}\right]\right)$ to

$\left[s_{0} t_{0}^{d}: s_{0} t_{0}^{d-1} t_{1}: \cdots: s_{0} t_{1}^{d}: s_{1} t_{0}^{d}: s_{1} t_{0}^{d-1} t_{1}: \cdots: s_{1} t_{0}^{d-e} t_{1}^{e}\right]$.

When $s_{0}=1$ and $t_{0}=1$, we recoup the first map.

Higher-dimensional rational normal scrolls are constructed in the same way. To obtain a variety of dimension $k$, there are $k$ rational normal curves in some $\mathbb{P}^{N}$ (whose spans do not intersect) and the scroll is swept out by the linear spaces spanned by the $k$ points on the curves for the same value of $t$.
More generally, a lattice polytope determines a monomial map by interpreting the lattice points lying in the polytope as exponent vectors. Toric geometry provides a dictionary between the properties of the polytopes and the properties of the (closure of the) image of the corresponding monomial map. A toric surface is a variety of minimal degree if and only if the polygon does not contain any lattice points in its interior. Excluding the polygons of the rational normal scrolls that have height one (like the one in Figure 3), there is only one more example: the triangle $\operatorname{conv}\{(0,0),(2,0),(0,2)\}$ corresponding to the Veronese surface $\nu_{2}\left(\mathbb{P}^{2}\right) \subset \mathbb{P}^{5}$.

Restricting a quadratic form to a rational normal scroll of dimension 2 yields an element in two variables that has degree 2 in $s$ and degree $2 d$ in $t$ (say $d \geqslant e$ ). In the higherdimensional cases, it is a form that has degree 2 in variables $s_{1}, s_{2}, \ldots, s_{k-1}$ and some degree $2 d$ in $t$. Homogenizing gives a form that is homogeneous of degree 2 in the $k$ variables $s_{0}, s_{1}, \ldots, s_{k-1}$ and homogeneous of degree $2 d$ in $t_{0}, t_{1}$. In [11], Man Duen Choi, T. Y. Lam, and Bruce Reznick called these elements biforms. A biform can also naturally be thought of as a symmetric matrix with real polynomial entries that is pointwise positive semidefinite on $\mathbb{P}^{1}$ as in [14].

From a historical perspective, Theorem 1.6 unites two fundamental results that developed independently in the 1880s. As we discuss the case of reducible varieties, we will see that a similar story repeated about 100 years later.

The reducible case. Thus far, we have emphasized sums of squares on irreducible varieties. However, Theorem 1.6 extends to all varieties including the reducible ones. In this subsection, we discuss some of the ideas involved in this generalization as well as some of its applications.

The varieties of minimal degree have the smallest possible degree among irreducible varieties $X \subset \mathbb{P}^{n}$ which span $\mathbb{P}^{n}$. In the reducible case, this inequality no longer holds (think of two skew lines in $\mathbb{P}^{3}$ ). The right generalization of this geometric concept is not immediately clear, but it turns out to be algebraic and involves syzygies. Consider homogeneous quadratic polynomials $f$ and $g$ in three variables defining two quadratic curves in $\mathbb{P}^{2}$. A syzygy is a linear relation between $f$ and $g$ where the coefficients are elements in the polynomial ring $\mathbb{R}\left[x_{0}, x_{1}, x_{2}\right]$. If at least one of $f$ or $g$ is irreducible, then every syzygy between them is generated by the obvious one: $(g) f+(-f) g=0$. When the polynomials $f$ and $g$ have a common linear factor, then a linear syzygy exists. For example, when $f=x_{0} x_{1}$ and $g=x_{0} x_{2}$, we have $\left(x_{2}\right) f+\left(-x_{1}\right) g=0$.

For a general set of polynomials, there can be syzygies among the syzygies. This data is usually organized into a minimal free resolution. One good way to gauge the complexity of such a resolution is called the CastelnuovoMumford regularity. A linear space has CastelnuovoMumford regularity 1 . Varieties of minimal degree have 
the smallest Castelnuovo-Mumford regularity among the varieties that are not linear spaces, namely 2 . For our purposes, 2-regular varieties are the right generalization for the concept of minimal degree. The next result first appeared as Theorem 9 in [6].

Theorem 1.7 (Blekherman, Sinn, and Velasco). Let $X$ be a nondegenerate totally-real projective variety in $\mathbb{P}^{n}$. Every nonnegative quadratic form on $X$ is a sum of squares, modulo the defining ideal of $X$, if and only if $X$ is a 2-regular variety.

What are all the 2-regular varieties? In 2006, David Eisenbud, Mark Green, Klaus Hulek, and Sorin Popescu gave a complete classification and a beautiful geometric characterization. A subvariety $X \subseteq \mathbb{P}^{n}$ is 2-regular if and only if, for any linear subspace $L$ such that $X \cap L$ is a finite set, this finite set is linearly independent (linear independence has to be defined carefully when the intersection is a nonreduced zero-dimensional scheme).

Instead of digging into the details of this classification, we concentrate on a special case, namely arrangements of coordinate subspaces. We consider projective varieties $X=\cup_{i=1}^{k} U_{i}$, where each $U_{i}$ is a linear subspace in $\mathbb{P}^{n-1}$ spanned by some set of standard coordinate vectors $e_{i}$, where $1 \leqslant i \leqslant n$. We also assume that corresponding homogeneous ideals are generated in degree 2 . For the combinatorially inclined, these subspace arrangements correspond to flag complexes. The definition ensures that each arrangement is determined by the coordinate lines in $\mathbb{P}^{n-1}$ that it contains.

Alternatively, each such arrangement $X$ of coordinate subspaces is determined by a graph. The graph has $n$ vertices corresponding to coordinates in $\mathbb{P}^{n-1}$. There is an edge between vertex $i$ and vertex $j$ when the coordinate subspace spanned by the $i$ th and $j$ th variables is contained in $X$. In other words, for each irreducible component $U_{i} \subset X$, we add the clique on all the vertices $j$ satisfying $e_{j} \in U_{i}$. Hence, the ideal defining the subvariety $X$ is generated by all the monomials $x_{i} x_{j}$ such that $\{i, j\}$ is not an edge in our graph; see Figure 4 . To stress that the subvariety $X$ comes from a graph $G$, we write $X(G)$.

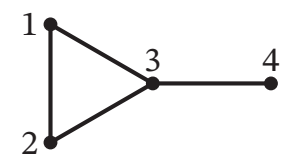

Figure 4. The graph corresponding to the subspace arrangement in $\mathbb{P}^{3}$ defined by the ideal $\langle x w, y w\rangle=\langle w\rangle \cap\langle x, y\rangle$.

The bijection between these subspace arrangements and graphs allows us to translate combinatorial properties of the graph $G$ into geometric properties of the subvariety $X(G)$. For instance, the dimension of $X(G)$ is one less than the clique number $\omega(G)$ (the size of the largest clique in $G)$. For which graphs $G$ is $X(G)$ a 2-regular variety?
Theorem 1.8 (Fröberg). The variety $X(G)$ is 2-regular if and only if the graph $G$ is chordal.

A graph $G$ is chordal if it contains no induced cycles of length at least four. In spite of their seemingly innocuous definition, chordal graphs are very useful and arise naturally in combinatorics, numerical linear algebra, and semidefinite programming; see [26]. Theorem 1.7 shows that chordal graphs determine the only varieties $X(G)$ on which nonnegative quadratic forms are sums of squares.

What does it mean for the quadratic form $q=x^{\top} A x$, where $x:=\left[\begin{array}{llll}x & y & z & w\end{array}\right]^{\top}$, to be nonnegative when restricted to $X(G)$ ? The restriction remembers certain minors of the matrix $A$, but does not know about other entries. For the graph in Figure 4, the restriction of the quadratic form $q$ to the 3-dimensional linear subspace defined by the ideal $\langle w\rangle$ corresponds to the upper left $(3 \times 3)$-submatrix in $A$. Similarly, the restriction of $q$ to the 2-dimensional linear subspace defined by the ideal $\langle x, y\rangle$ corresponds to the lower right $(2 \times 2)$-submatrix in $A$. Hence, the restriction has forgotten about the entries $a_{1,4}$ and $a_{2,4}$ in $A$. In this sense, the restriction of the quadratic form $q$ to subspace arrangment $X(G)$ is a partially specified real symmetric matrix. Nonnegativity of the restriction means that the corresponding submatrices of $A$ are positive semidefinite.

What does it mean for a nonnegative quadratic form on $X(G)$ to be a sum of squares? Our description of the ideal defining $X(G)$ implies that restricting the quadratic form $x^{\top} A x$ to subvariety $X(G)$ is the same as erasing the entries of $A$ corresponding to nonedges of $G$. To write a quadratic form as a sum of squares modulo the ideal of $X$ is tantamount to choosing the unspecified entries of the matrix so that the entire symmetric matrix $A$ is positive semidefinite. This problem is known as a positive semidefinite matrix completion problem. It is well studied with applications in combinatorics, discrete geometry, and statistics.

We can now reinterpret the equivalence between the chordality of $G$ and $P_{X(G)}=\Sigma_{X(G)}$ in terms of matrix completion, reproving the following theorem.

Theorem 1.9 (Grone, Johnson, Sá, and Wolkowicz). Let $X(G)$ be the subspace arrangement associated to a graph $G$. Every nonnegative quadratic form on $X(G)$ is a sum of squares, modulo the ideal of $X(G)$, if and only if the graph $G$ is chordal.

Remarkably, Theorem 1.7 proves that Theorems 1.8 and 1.9 are in fact equivalent suggesting a link between matrix completion problems and commutative algebra.

\section{Pythagoras Numbers}

In 1770, Joseph-Louis Lagrange proved his four-squares theorem: every nonnegative integer can be represented as a sum of four integer squares. Adrien-Marie Legendre extended this result in 1797 or 1798 , proving that three squares suffice unless the integer has the form $4^{k}(8 m+7)$ 
for some nonnegative integers $k$ and $m$. Pierre de Fermat had already determined in 1625 the exact conditions for a nonnegative integer to be expressible as a sum of two squares. Altogether this work gives a relatively complete description of the sum-of-squares expressions for integers. This achievement encourages one to look for analogues for sums of squares in other rings. This section explores this line of inquiry for homogeneous coordinate rings of projective real subvarieties.

Moving away from the relation between nonnegative elements and sums of squares, we endeavor to stratify the various sum-of-squares decompositions of an element according to their size. The length of an element $f$ in the homogeneous coordinate ring of a projective subvariety is the smallest number of summands needed to express $f$ as a sum of squares.

Example 2.1. The binary quartic $f:=\left(t_{0}^{2}\right)^{2}+\left(2 t_{1}^{2}\right)^{2}$ has length two. It has a second representation as the sum of two squares, namely $f=\left(t_{0}^{2}-2 t_{1}^{2}\right)^{2}+\left(2 t_{0} t_{1}\right)^{2}$. Taking the average of these expressions, we obtain

$$
f=\left(\frac{1}{\sqrt{2}} t_{0}^{2}\right)^{2}+\left(\sqrt{2} t_{1}^{2}\right)^{2}+\left(\frac{1}{\sqrt{2}} t_{0}^{2}-\sqrt{2} t_{1}^{2}\right)^{2}+\left(\sqrt{2} t_{0} t_{1}\right)^{2},
$$

and deduce that $f=(B t)^{\top}(B t)$, where

$$
B:=\frac{1}{\sqrt{2}}\left[\begin{array}{rrr}
1 & 0 & 0 \\
0 & 2 & 0 \\
1 & -2 & 0 \\
0 & 0 & 2
\end{array}\right] \quad \text { and } \quad t:=\left[\begin{array}{c}
t_{0}^{2} \\
t_{1}^{2} \\
t_{0} t_{1}
\end{array}\right] .
$$

Under the orthogonal change of coordinates given by

$$
Q:=\frac{1}{6}\left[\begin{array}{rrrr}
3 \sqrt{2} & 0 & 3 \sqrt{2} & 0 \\
0 & 0 & 0 & 6 \\
\sqrt{6} & 2 \sqrt{6} & -\sqrt{6} & 0 \\
2 \sqrt{3} & -2 \sqrt{3} & -2 \sqrt{3} & 0
\end{array}\right],
$$

this sum of four squares becomes a sum of three squares: $f=(Q B t)^{\top}(Q B t)=\left(t_{0}^{2}-t_{1}^{2}\right)^{2}+\left(\sqrt{2} t_{0} t_{1}\right)^{2}+\left(\sqrt{3} t_{1}^{2}\right)^{2}$.

Sum-of-squares representations are parametrized by a convex semialgebraic set. The Gram spectrahedron of an element $f$ in the homogeneous coordinate ring of a real subvariety $X \subseteq \mathbb{P}^{n}$ consists of all positive semidefinite quadratic forms in $\mathbb{R}\left[x_{0}, x_{1}, \ldots, x_{n}\right]$ that restrict to $f$. In Example 2.1, the subvariety $X=v_{2}\left(\mathbb{P}^{1}\right)$ is a conic in $\mathbb{P}^{2}$ and the Gram spectrahedron of the binary quartic is a line segment: the convex hull of the two quadratic forms of rank 2 whose restriction to $\nu_{2}\left(\mathbb{P}^{1}\right)$ is $f$. The Gram spectrahedra of binary forms grow more interesting as the degree increases. For binary sextics, Figure 5 visualizes the Zariski closure of the boundary of this 3-dimensional Gram spectrahedron. In this case the boundary lies on a Kummer surface and the spectrahedron is the convex region containing four nodes; see Subsection 4.2 in [12].

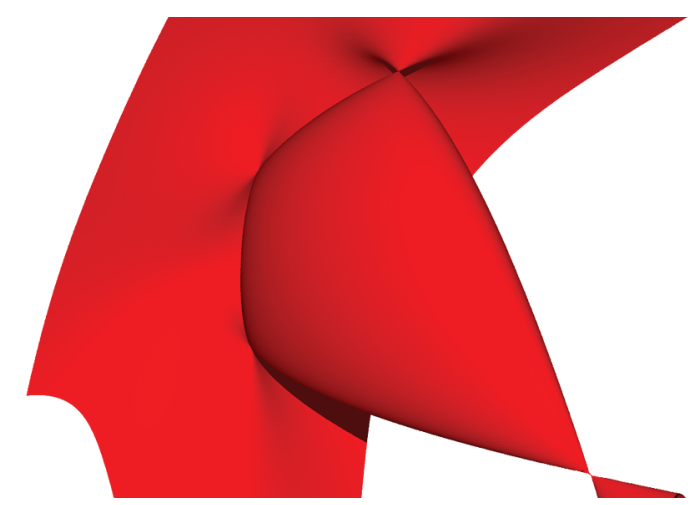

Figure 5. The Gram spectrahedron of a binary sextic.

To see some repercussions of working modulo the ideal of a subvariety on length, let $X$ be the hypersurface in $\mathbb{P}^{3}$ defined by $x^{2}+y^{2}+z^{2}-w^{2}=0$. Consider the quadratic element in the homogeneous coordinate ring of $X$ determined by $f=2\left(x^{2}-y z\right)+w^{2}$. Despite $f$ having a negative sign when evaluated at some points on $\mathbb{P}^{3}$, the corresponding element on $X$ is a sum of squares; homogenizing the first equation in this article gives

$$
2\left(x^{2}-y z\right)+w^{2}+\left(x^{2}+y^{2}+z^{2}-w^{2}\right)=3 x^{2}+(y-z)^{2} .
$$

What is the length of $f$ on $X$ ? The element $f$ vanishes at the real point $p:=[0: 1: 1: \sqrt{2}] \in X$. Since a sumof-squares representation of $f$ on $X$ evaluated at the point $p$ is a sum of nonnegative real numbers adding to 0 , every summand also vanishes at the point $p$. It follows that every sum-of-squares representation $f$ on $X$ is a sum of squares of linear forms vanishing at $p$. In more geometric terms, the representation for $f$ on $X$ factors through a projection. The projection away from the point $p$ is the rational map $\pi_{p}: \mathbb{P}^{3} \rightarrow \mathbb{P}^{2}$ whose components are given by a basis of the linear forms vanishing at $p$ such as

$$
\pi_{p}([x: y: z: w])=[x: y-z: \sqrt{2} y-w] .
$$

Writing $\mathbb{R}\left[x_{0}, x_{1}, x_{2}\right]$ for the homogeneous coordinate ring of $\pi_{p}(X)=\mathbb{P}^{2}$, the element $f$ corresponds to $2 x_{0}^{2}+x_{1}^{2}$. We conclude that $f$ has length at least two on $X$.

To control the length of all elements, we introduce a new invariant. For any real subvariety $X \subseteq \mathbb{P}^{n}$, the Pythagoras number $\operatorname{py}(X)$ is defined to be the smallest nonnegative integer $r$ such that any sum of squares of linear elements in the homogeneous coordinate ring of $X$ can be expressed as the sum of at most $r$ squares. Although not apparent from the definition, this semialgebraic invariant reflects the underlying geometry of the complex points in $X$. For instance, we will see that having a small Pythagoras number characterizes varieties of minimal degree. Additionally, this numerical invariant is a crucial parameter in non-convex approaches to semidefinite optimization, leading to valuable guarantees for sum-of-squares methods on varieties; see [10]. Before delving into these results, we revisit some examples from the previous section. 
The simplest situation occurs when $X=\mathbb{P}^{n}$. For any quadratic form $f$ on $\mathbb{P}^{n}$, there is a unique real symmetric matrix $A$ such that $f=x^{\top} A x$, where $x:=\left[\begin{array}{llll}x_{0} & x_{1} & \cdots & x_{n}\end{array}\right]^{\top}$. The finite-dimensional spectral theorem establishes that the matrix $A$ is orthogonally diagonalizable. This means that there is an orthogonal matrix $Q$ such that $y:=Q x$ and $f=\sum_{i=0}^{n} \lambda_{i} y_{i}^{2}$, where the real numbers $\lambda_{0}, \lambda_{1}, \ldots, \lambda_{n}$ are the eigenvalues of $A$. The quadratic form $f$ is nonnegative if and only if these eigenvalues are nonnegative and have real square roots. Thus, we may pull the coefficients inside the squares and obtain an expression for $f$ as a sum of squares. The number of squares in this expression equals the number of nonzero eigenvalues, so the length of the quadratic form $f$ is bounded above by the rank of $A$. Conversely, if $f=\left(v_{1}^{\top} x\right)^{2}+\left(v_{2}^{\top} x\right)^{2}+\cdots+\left(v_{k}^{\top} x\right)^{2}$ is a sum of squares of linear forms whose coefficient vectors are $v_{1}, v_{2}, \ldots, v_{k} \in \mathbb{R}^{n+1}$, then the corresponding matrix $A=v_{1} v_{1}^{\top}+v_{2} v_{2}^{\top}+\cdots+v_{k} v_{k}^{\top}$ is a sum of $k$ matrices, each having rank 1 . Thus, the matrix $A$ has rank at most $k$. We surmise that the length of $f$ is equal to the rank of $A$, which proves that py $\left(\mathbb{P}^{n}\right)=n+1$. The same reasoning implies that $\operatorname{py}(X)=n+1$ whenever the defining ideal of the real subvariety $X \subseteq \mathbb{P}^{n}$ contains no polynomials of degree less than 3 .

Another situation is easy to understand. Consider a nonnegative bivariate form $f$ of degree $2 j$, corresponding to a quadratic form on $v_{j}\left(\mathbb{P}^{1}\right)$. Dehomogenizing $f$ by setting $t_{1}=1$ yields a univariate polynomial $q \in \mathbb{R}\left[t_{0}\right]$. Since the coefficients of $\hat{f}$ are real, any complex roots come in conjugate pairs. It follows that $f$ factors over the complex numbers as $f=q_{0} q_{1} \overline{q_{1}}$, where $q_{0}$ is the product of the linear forms corresponding to real roots of $\hat{f}, q_{1}$ is the product of a linear form associated to each pair of complex roots of $\hat{f}$, and $\overline{q_{1}}$ is the complex conjugate of $q_{1}$. Since $f$ is nonnegative, every real root has even multiplicity, so $q_{0}$ is automatically a square. From the identity $q_{1} \overline{q_{1}}=\operatorname{Re}\left(q_{1}\right)^{2}+\operatorname{Im}\left(q_{1}\right)^{2}$, we see that $f$ is a polynomial sum of two squares. In terms of Pythagoras numbers, this shows $\operatorname{py}\left(\nu_{j}\left(\mathbb{P}^{1}\right)\right)=2$ for all $j \geqslant 2$.

In our third situation, we focus on the appearance of Pythagoras numbers in matrix completion problems. As in the previous section, fix a pattern of specified and unspecified off-diagonal entries in a matrix. This data determines a graph $G$ or square-free quadratic monomial ideal, whose associated variety $X(G)$ is a coordinate subspace arrangement. Rather than looking for conditions that make a partially-specified matrix completable to a positive semidefinite matrix, we now ask for low rank completions. Given a matrix with missing entries that is known to have a positive semidefinite completion, what is the lowest rank among its completions? The smallest nonnegative integer $r$, such that any completable partially-specified matrix can be completed to a positive semidefinite matrix of rank $r$, is precisely the Pythagoras number of variety $X(G)$. In this context, the Pythagoras number was called the Gram dimension by Monique Laurent and Antonios Varvitsiotis in [19]. It has applications in distance realization problems and rigidity theory.

Calculating Pythagoras numbers for the Veronese embeddings of projective space is still an open problem. For all positive integers $d$ and $n$, what is $\operatorname{py}\left(\nu_{d}\left(\mathbb{P}^{n}\right)\right)$ ? To date, the strongest results are due to Claus Scheiderer [24]. He proves that $\operatorname{py}\left(\nu_{d}\left(\mathbb{P}^{2}\right)\right) \in\{d+1, d+2\}$. Assuming the validity of conjectures by Anthony Iarrobino and Vassil Kanev on the Hilbert function of ideals of finite sets of points [18], he also proves that the asymptotic growth rate is $\operatorname{py}\left(v_{d}\left(\mathbb{P}^{n}\right)\right) \in O\left(d^{n / 2}\right)$. Our geometric perspective provides a systematic method for bounding Pythagoras numbers. The next lemma is a first step in this direction.

Lemma 2.2. Let $X \subseteq \mathbb{P}^{n}$ be a totally-real subvariety.

(i) For any totally-real subvariety $Y \subseteq \mathbb{P}^{n}$ such that $X \subseteq Y$, we have the inequality $\operatorname{py}(X) \leqslant \operatorname{py}(Y)$.

(ii) If $\pi_{p}: \mathbb{P}^{n} \rightarrow \mathbb{P}^{n-1}$ denotes the projection away from $a$ real point $p \in X$, then we have $\operatorname{py}(X) \geqslant \operatorname{py}\left(\pi_{p}(X)\right)$. More precisely, the length of any quadratic element $f$ on $X$ that vanishes at the point $p$ is bounded below by the length of an element $g$ on $Y$ satisfying $f=\pi_{p}^{*}(g)$.

Part (ii) showcases the importance of convexity. By considering all elements that vanish at a fixed real point $p \in X$, we identify a face $F$ of the sum-of-squares cone $\Sigma_{X}$. For any element $f$ lying on the face $F$, every summand in any expression for $f$ as a sum of squares must also lie on $F$. It follows that the length of an element in $F$ is determined by the face alone. Moreover, the face $F$ of $\Sigma_{X}$ specified by evaluation at the point $p \in X$ is isomorphic to the cone $\Sigma_{Z}$, where $Z:=\pi_{p}(X)$. Hence, the Pythagoras number of $X$ is bounded below by the Pythagoras number of $\pi_{p}(X)$.

To estimate the Pythagoras number of a totally-real subvariety $X \subseteq \mathbb{P}^{n}$, Lemma 2.2 suggests that we recognize a simpler subvariety containing $X$ or successively project away from points to obtain a simpler variety. For either approach to work, we need a sufficiently large class of varieties having a known Pythagoras number. Corollary 32 in [6] and Theorem 2.1 in [5] demonstrate that varieties of minimal degree and their reducible generalizations, called 2-regular varieties, form such a class of varieties.

Theorem 2.3 (Blekherman, Sinn, and Velasco). Let $X$ be a nondegenerate totally-real 2-regular subvariety in $\mathbb{P}^{n}$. We have $\operatorname{py}(X)=\operatorname{dim}(X)+1$. When $X$ is irreducible, we have $\operatorname{py}(X)=\operatorname{dim}(X)+1$ if and only if $X$ is a variety of minimal degree.

We may bound the Pythagoras number of a totally-real subvariety by embedding it in a 2-regular variety.

This geometric approach applies to matrix completion problems. Recall that a 2-regular quadratic square-free 
monomial ideal corresponds to a chordal graph $H$ and the dimension of the associated variety $X(H)$ is equal to the size of the largest clique of $H$ minus one. By considering all chordal graphs that contain a given graph $G$, we have $\operatorname{py}(X(G)) \leqslant \operatorname{tw}(G)+1$, where $\operatorname{tw}(G)$ is the tree-width of graph $G$; as in [19], the tree-width is defined to be one less than the minimum clique number among all chordal graphs containing $G$.

Quadratic persistence. As we have already observed, the equation $\operatorname{py}(Z)=m+1$ holds whenever the defining ideal of the real subvariety $Z \subseteq \mathbb{P}^{m}$ contains no polynomials of degree less than 3. Using this observation and Lemma 2.2, we may bound the Pythagoras number of a totally-real subvariety $X \subseteq \mathbb{P}^{n}$ by successively projecting it away from $k$ real points so that no quadric polynomial vanishes on the image variety $Z \subseteq \mathbb{P}^{n-k}$. If it is possible to eliminate all quadrics using $k$ projections, then one would obtain $\operatorname{py}(X) \geqslant \operatorname{py}(Z)=n-k+1$. The cardinality of a smallest set of points on $X$ which, via projection, eliminate all quadrics is called the quadratic persistence of $X$ and denoted $\mathrm{qp}(X)$. When $X$ is irreducible, choosing points at random will always suffice. This numerical invariant is purely algebraic because it measures vanishing of quadratic forms (including the complex quadratic forms in the ideal of $X$ ) on sets of points on the variety.

To become more acquainted with this process, Table 1 catalogues the dimension of the vector space of quadric polynomials vanishing on various projections of $v_{d}\left(\mathbb{P}^{n}\right)$ for small values of $d$ and $n$. The $k$ th row displays the dimension of the vector space of quadrics vanishing in the projection away from $k$ generic points. This table shows

Table 1. Dimensions of the space quadrics.

\begin{tabular}{cccc} 
\# Points & $\nu_{2}\left(\mathbb{P}^{2}\right) \subset \mathbb{P}^{5}$ & $\nu_{3}\left(\mathbb{P}^{2}\right) \subset \mathbb{P}^{9}$ & $\nu_{3}\left(\mathbb{P}^{3}\right) \subset \mathbb{P}^{19}$ \\
\hline 0 & 6 & 27 & 126 \\
1 & 3 & 20 & 110 \\
2 & 1 & 14 & 95 \\
3 & 0 & 9 & 81 \\
4 & 0 & 5 & 68 \\
5 & 0 & 2 & 56 \\
6 & 0 & 0 & 45
\end{tabular}

that quadratic persistences of the varieties in the second and third columns are equal to 3 and 6 , respectively. It follows that $\operatorname{py}\left(\nu_{2}\left(\mathbb{P}^{2}\right)\right) \geqslant 3$ and $\operatorname{py}\left(\nu_{3}\left(\mathbb{P}^{2}\right)\right) \geqslant 4$. Both of these inequalities are, in fact, sharp. The last column only shows that $\mathrm{qp}\left(\nu_{3}\left(\mathbb{P}^{3}\right)\right) \geqslant 7$.

How many quadrics do we lose in each step? It is straightforward to show that, in each projection step, the number of quadrics that disappear from the ideal is at most the codimension of the variety. Surprisingly, this inequality is an equality for each entry in Table 1 . If this pattern were to continue, then we would deduce that
$\mathrm{qp}\left(\nu_{3}\left(\mathbb{P}^{3}\right)\right)=12$. The Iarrobino-Kanev conjectures mentioned earlier imply that the number of quadrics lost at every step is maximal for all values of $n$ and $d$. Thus, they imply $\mathrm{qp}\left(\nu_{3}\left(\mathbb{P}^{3}\right)\right)=12$.

More generally, for an irreducible subvariety $X \subseteq \mathbb{P}^{n}$, the projection away from a set of $\operatorname{codim}(X)$ generic points maps $X$ surjectively onto $\mathbb{P}^{\operatorname{dim}(X)}$. Hence, there are no quadric polynomials vanishing on the image. It follows that $\mathrm{qp}(X) \leqslant \operatorname{codim}(X)$ and $\mathrm{py}(X) \geqslant \operatorname{dim}(X)+1$.

Quadratic persistence depends only on the complex algebraic geometry of a variety $X$. It is an algebraic invariant rather than a semialgebraic one, so tools from commutative and homological algebra apply. Describing this in detail would take us too far afield. Nevertheless, we want to state two consequences of these tools, namely the characterizations (modulo a few technical assumptions) of all irreducible varieties with minimal and next-to-minimal Pythagoras numbers. The following theorems appear as Theorems 1.4 and 1.5 in [7].

Theorem 2.4 (Blekherman, Smith, Sinn, and Velasco). Let $X \subseteq \mathbb{P}^{n}$ be a nondegenerate totally-real irreducible subvariety. The following three conditions are equivalent:

(1) $\operatorname{py}(X)=\operatorname{dim}(X)+1$,

(2) $\operatorname{deg}(X)=\operatorname{codim}(X)+1$,

(3) $\mathrm{qp}(X)=\operatorname{codim}(X)$.

Theorem 2.5 (Blekherman, Smith, Sinn, and Velasco). Let $X \subseteq \mathbb{P}^{n}$ be a nondegenerate totally-real irreducible subvariety. If $X$ is arithmetically Cohen-Macaulay, then the following three conditions are equivalent:

(1) $\operatorname{py}(X)=\operatorname{dim}(X)+2$,

(2) $\operatorname{deg}(X)=\operatorname{codim}(X)+2$ or $X$ is a subvariety having codimension 1 in a variety of minimal degree,

(3) $\mathrm{qp}(X)=\operatorname{codim}(X)-1$.

In all these cases and in all cases that we understand, quadratic persistence detects the Pythagoras number: $\mathrm{qp}(X)+\mathrm{py}(X)=n+1$. We have no reason to expect this behavior, so it would be interesting to find an example where this equation does not hold.

\section{Sum-of-Squares Multipliers}

Knowing that there exist nonnegative polynomials that cannot be represented as a polynomial sum of squares, we want other effective ways of recognizing nonnegativity. The 17th problem on Hilbert's celebrated list proposes a candidate. Hilbert asks whether every polynomial that is nonnegative, when regarded as a function from $\mathbb{R}^{n}$ to $\mathbb{R}$, equals a sum of squares of rational functions.

Emil Artin [1] solved this problem in 1927. Before stating this result, observe that the intermediate value theorem implies that every real polynomial having odd degree is negative at some point in $\mathbb{R}^{n}$. Moreover, the homogenization of a polynomial takes only nonnegative values if 
and only if the same is true for the dehomogenized polynomial. Hence, we may restrict our attention to homogeneous polynomials of even degree.

Given a nonnegative polynomial $f$, Artin demonstrates that, for some positive integer $r$, there exist polynomials $g_{1}, g_{2}, \ldots, g_{r}, h_{1}, h_{2}, \ldots, h_{r}$ such that

$$
f=\left(\frac{h_{1}}{g_{1}}\right)^{2}+\left(\frac{h_{2}}{g_{2}}\right)^{2}+\cdots+\left(\frac{h_{r}}{g_{r}}\right)^{2} .
$$

For instance, the Motzkin polynomial is equal to a sum of four squares of rational functions:

$$
\begin{aligned}
& x_{0}^{6}+x_{1}^{4} x_{2}^{2}+x_{1}^{2} x_{2}^{4}-3 x_{0}^{2} x_{1}^{2} x_{2}^{2} \\
= & \left(\frac{x_{0} x_{1} x_{2}\left(x_{1}^{2}+x_{2}^{2}-2 x_{0}^{2}\right)}{x_{1}^{2}+x_{2}^{2}}\right)^{2}+\left(\frac{x_{1}^{2} x_{2}\left(x_{1}^{2}+x_{2}^{2}-2 x_{0}^{2}\right)}{x_{1}^{2}+x_{2}^{2}}\right)^{2} \\
& +\left(\frac{x_{1} x_{2}^{2}\left(x_{1}^{2}+x_{2}^{2}-2 x_{0}^{2}\right)}{x_{1}^{2}+x_{2}^{2}}\right)^{2}+\left(\frac{x_{0}^{3}\left(x_{1}^{2}-x_{2}^{2}\right)}{x_{1}^{2}+x_{2}^{2}}\right)^{2} .
\end{aligned}
$$

Artin's original solution comes from studying sums of squares in arbitrary fields. The key insight uses total orderings that are compatible with field operations to characterize the subset of elements in a field that can be expressed as a sum of squares. Although profoundly influential in the theory of real-closed fields, this nonconstructive proof does not bound the number of squares or the degrees of the polynomials appearing in the rational functions. Albrecht Pfister [22] subsequently proved that, for a homogeneous polynomial in $n$ variables, $2^{n}$ squares suffice.

In our search for constructive methods of identifying nonnegative polynomials, we prefer a slightly different formulation. We abandon the rational functions by finding a common denominator. Given a homogeneous polynomial $f$ that is nonnegative on $\mathbb{R}^{n}$, it follows that, for some positive integers $s$ and $r$, there are homogeneous polynomials $g_{1}, g_{2}, \ldots, g_{s}, h_{1}, h_{2}, \ldots, h_{r}$ such that

$$
\left(g_{1}^{2}+g_{2}^{2}+\cdots+g_{s}^{2}\right) f=h_{1}^{2}+h_{2}^{2}+\cdots+h_{r}^{2} .
$$

Allowing the multiplier $g:=g_{1}^{2}+g_{2}^{2}+\cdots+g_{s}^{2}$ to be any polynomial sum of squares rather than just the square of a single homogeneous polynomial enlarges the pool of potential certificates. At the expense of doubling the degree of the multiplier and, at worst, replacing $r$ by $r s$, we recover a representation of $f$ as a sum of squares of rational functions from $g^{2} f=g\left(h_{1}^{2}+h_{2}^{2}+\cdots+h_{r}^{2}\right)$. To effectively certify nonnegativity, one seeks to bound the degree on a multiplier $g$.

An explicit bound on multipliers is a recent discovery. Henri Lombardi, Daniel Perrucci, and Marie-Françoise Roy [20] prove that, for any nonnegative polynomial $f$ of degree $d$ in $n$ variables, there exists a sum-of-squares multiplier $g$ of degree less than

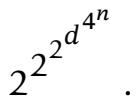

This tower of five exponents arises from bounding the complexity of a quantifier elimination problem. Roughly speaking, a cylindrical algebraic decomposition produces two levels, a constructive version of the fundamental theorem of algebra gives one level, and constructions based on the intermediate value theorem are responsible for the other two levels in the tower.

In contrast, very little is known about worst-case lower bounds on the degree of a multiplier. Grigoriy Blekherman, João Gouveia, and James Pfeiffer [2] establish that, for all positive integers $n$, there is a nonnegative quartic polynomial $f$ in $n$ variables such that any sum-of-squares multiplier $g$ must have degree at least $n$. Thus, we see that there is an embarrassingly large gap between the current upper and lower bounds on the degree of a multiplier. In an attempt to redress this issue, we aim to provide much tighter degree bounds in some situations. We again appeal to complex projective geometry.

Unlike in the previous sections, we do not limit ourselves to quadratic functions on a real subvariety $X \subseteq \mathbb{P}^{n}$. As before, let $R$ be the homogeneous coordinate ring of $X$. For any nonnegative integer $j$, let $P_{X, 2 j}$ denote the set of homogeneous elements in $R$ of degree $2 j$ that are nonnegative on $X$. Similarly, let $\Sigma_{X, 2 j}$ denote the set of homogeneous elements in $R$ of degree $2 j$ that are sums of squares. Compared with our earlier notation, $P_{X, 2 j}$ and $\Sigma_{X, 2 j}$ are the same as $P_{\nu_{j}(X)}$ and $\Sigma_{\nu_{j}(X)}$. Hence, both $P_{X, 2 j}$ and $\Sigma_{X, 2 j}$ are full-dimensional convex cones in the real vector space $R_{2 j}$ that contain no line and are closed in the Euclidean topology. Considering higher-degree elements in $R$ seems more natural for multipliers.

The existence of a nonnegative multiplier $g$ such that the product $g f$ is a sum of squares manifestly confirms that the element $f$ is nonnegative when evaluated at any point for which $g$ does not vanish. The consequences for other points are not immediately as clear. When the complement of this vanishing set is dense in the Euclidean topology, it follows that the element $f$ is nonnegative at every point. This rationale always applies to polynomials on $\mathbb{R}^{n}$, but becomes more complicated when the algebraic variety $X$ is reducible or singular.

For real projective curves, we produce sharp degree bounds on sum-of-squares multipliers in terms of three fundamental numerical invariants. As in Section 1, the degree of the curve $X \subset \mathbb{P}^{n}$ is the number of points in the intersection of the curve and a general $(n-1)$-dimensional linear subspace. The (arithmetic) genus of a projective curve $X$ is denoted by $\mathrm{p}_{\mathrm{a}}(X)$. When $X$ is nonsingular over $\mathbb{C}$, this complex curve may be viewed as a Riemann surface and this numerical invariant coincides with its topological genus. Our third invariant $\mathrm{m}(X)$ is the smallest integer $j$ such that the Hilbert function and the Hilbert polynomial of $X$ agree when evaluated at any integer greater than 
or equal to $j$. Using these invariants, Theorem 1.1 in [7] gives the following bounds.

Theorem 3.1 (Blekherman, Smith, and Velasco). For any nondegenerate totally-real projective curve $X \subset \mathbb{P}^{n}$, any nonnegative element $f \in P_{X, 2 j}$, and all nonnegative integers $k$ satisfying $k \geqslant \max \left\{2 \mathrm{p}_{\mathrm{a}}(X) / \operatorname{deg}(X), \mathrm{m}(X)\right\}$, there exists a nonzero sum of squares $g \in \Sigma_{X, 2 k}$ such that the product $g f \in \Sigma_{2(j+k)}$ is also a sum of squares.

Conversely, for all integers $n$ and $j$ greater than 1 , there exist a totally-real smooth curve $X \subset \mathbb{P}^{n}$ and a nonnegative element $f \in P_{X, 2 j}$ such that, for all nonnegative integers $k$ satisfying $k<$ $\max \left\{2 \mathrm{p}_{\mathrm{a}}(X) / \operatorname{deg}(X), \mathrm{m}(X)\right\}$ and all nonzero sum of squares $g \in \Sigma_{X, 2 k}$, the product $g f \notin \Sigma_{X, 2(j+k)}$ is not a sum of squares.

The uniform degree bound on the multiplier $g$ in the first part of Theorem 3.1 is determined by just the complex geometry of the curve $X$. It is, notably, independent of both the degree of the nonnegative element $f$ and the topology of the real points in $X$.

Example 3.2. Consider a totally-real complete intersection curve $X \subset \mathbb{P}^{n}$ cut out by homogeneous polynomials of degrees $d_{1}, d_{2}, \ldots, d_{n-1}$. The numerical invariants of interest are $\operatorname{deg}(X)=d_{1} d_{2} \cdots d_{n-1}$,

$$
\mathrm{p}_{\mathrm{a}}(X)=\frac{1}{2} d_{1} d_{2} \cdots d_{n-1}\left(d_{1}+d_{2}+\cdots+d_{n-1}-n-1\right)+1,
$$

and $\mathrm{m}(X)=d_{1}+d_{2}+\cdots+d_{n-1}-n-1$. Theorem 3.1 shows that, for all $k \geqslant d_{1}+d_{2}+\cdots+d_{n-1}-n$ and all nonnegative elements $f \in P_{X, 2 j}$, there is a nonzero sum of squares $g \in \Sigma_{X, 2 k}$ such that the product $g f \in \Sigma_{X, 2(j+k)}$ is a sum of squares.

Example 3.3. Theorem 3.1 is not sharp on every curve. Let $X$ be the image of the map $\mathbb{P}^{1} \rightarrow \mathbb{P}^{2}$ defined by $\left[t_{0}: t_{1}\right] \mapsto$ $\left[t_{0}^{2} t_{1}\left(t_{0}-t_{1}\right): t_{0} t_{1}^{2}\left(t_{0}-t_{1}\right): t_{0}^{4}+t_{1}^{4}\right]$. This planar curve has degree 4 , arithmetic genus 3 , and $\mathrm{m}(X)=2$, so Theorem 3.1 would require $k \geqslant 2$. However, one can prove that, for all nonnegative elements $f \in P_{X, 2}$, there exists a nonzero sum of squares $g \in \Sigma_{X, 2}$ such that the product $g f \in \Sigma_{X, 4}$ is a sum of squares. For more information about this analysis, see Example 5.3 in [7].

The proofs for the two parts of Theorem 3.1 are disjoint. The upper bound on the minimum degree of a multiplier is derived from a new Bertini theorem in convex algebraic geometry and the lower bound is obtained by deforming rational Harnack curves on toric surfaces.

For the first part, we reinterpret the nonexistence of a sum-of-squares multiplier $g \in \Sigma_{X, 2 k}$ as asserting that the convex cones $\Sigma_{X, 2 j+2 k}$ and $f \cdot \Sigma_{X, 2 k}$ intersect only at zero. If a real subvariety $X \subseteq \mathbb{P}^{n}$ has a linear functional separating these cones, then we show that a sufficiently general hypersurface section of $X$ also does. The phrase "sufficiently general" means belonging to a nonempty open subset in the
Euclidean topology of the relevant parameter space. Unexpectedly, this convex variant of the Bertini Theorem relies on a characterization of spectrahedra that has many facets in a neighborhood of every point. Recognizing this dependency is the main insight. By repeated applications of our Bertini theorem, we reduce to the case of points.

For the second part, we establish that having a nonnegative element vanish at a relatively large number of isolated real singularities prevents it from having a low-degree sum-of-squares multiplier. The hypotheses needed to realize this basic premise are formidable. Nonetheless, this transforms the problem into finding enough curves that satisfy the conditions and maximizing the number of isolated real singularities. Harnack curves having the maximal number of connected real components in a prescribed topological arrangement are natural contenders. We confirm that rational singular Harnack curves on toric surfaces fulfill the technical requirements. By perturbing both the curves $X$ and the nonnegative element $f \in R_{2 j}$, we exhibit smooth curves and nonnegative elements without low-degree sum-of-squares multipliers. Miraculously, for totally-real projective curves, the upper bounds and lower bounds on the degree coincide.

This approach also works for surfaces of minimal degree. Recall that a subvariety $X \subset \mathbb{P}^{n}$ has minimal degree if it is nondegenerate and $\operatorname{deg}(X)=1+\operatorname{codim}(X)$. Even when $X$ is a variety of minimal degree, its $j$ th Veronese embedding $\nu_{j}(X)$ rarely is. Hence, multipliers are needed to certify nonnegativity. Theorem 1.2 in [7] provides the following bounds.

Theorem 3.4 (Blekherman, Smith, and Velasco). Let $X$ be a totally-real surface of minimal degree in $\mathbb{P}^{n}$. For any nonnegative element $f \in P_{X, 2 j}$, there exists a nonzero sum of squares $g \in \Sigma_{X, j^{2}-j}$ such that the product $g f \in \Sigma_{X, j^{2}+j}$ is a sum of squares.

Conversely, for any integer $j$ greater than 4 , there exists a nonnegative element $f \in P_{X, 2 j}$ such that, for any positive integer $k$ satisfying $k<j-2$ and any nonzero sum of squares $g \in \Sigma_{X, 2 k}$, the product $g f \notin \Sigma_{X, 2(j+k)}$ is not a sum of squares.

Unlike for curves, Theorem 3.4 demonstrates that the minimum degree of a multiplier $g$ generally depends on the degree of the nonnegative element $f$. Furthermore, our techniques do not typically yield sharp bounds.

Even in the special case $X=\mathbb{P}^{2}$, our geometric outlook leads to something new. For example, we reprove and prove the following two results for ternary octics:

- For all nonnegative elements $f \in P_{P^{2}, 8}$, there exists a nonzero sum of squares $g \in \Sigma_{\mathbb{P} 2,4}$ such that the product $g f \in \Sigma_{\mathbb{P} 2}, 12$ is also a sum of squares.

- There exists a nonnegative element $f \in P_{\mathbb{P}^{2}, 8}$ such that, for all nonzero sum of squares $g \in \Sigma_{\mathbb{P}^{2}, 2}$, the product $g f \notin \Sigma_{\mathbb{P} 2}, 10$ is not a sum of squares. 
Together these observations give the first tight bounds on the degrees of sum-of-squares multipliers for polynomials since Hilbert's 1893 paper [16] where he establishes sharp bounds for ternary sextics.

\section{Continuing the Story}

This is not the end of the story. We have no doubt that many fascinating new chapters about the connections between complex algebraic geometry and nonnegativity have yet to be written. In particular, we would like to see solutions to the following open problems.

Quantifying the discrepancy: How should one measure the difference between the nonnegative cone $P_{X}$ and the sum-of-squares cone $\Sigma_{X}$ when the subvariety $X \subset \mathbb{P}^{n}$ is not of minimal degree? For some estimates on their relative volumes, see Chapter 4 in [4].

Pythagoras numbers: Can one clarify the relation between quadratic persistence and the Pythagoras number? For instance, is the classification of the real subvarieties $X \subset \mathbb{P}^{n}$ satisfying $\mathrm{qp}(X)+\operatorname{py}(X)=n+1$ in sight?

Sharp degree bounds: Which geometric invariants of the associated complex projective variety determine the smallest degree of a sum-of-squares multiplier on a surface or higher-dimensional variety?

We recommend the books [4] and [21] to readers interested in further exploring these questions. The authors thank the SIAM Activity Group on Algebraic Geometry whose welcoming and interdisciplinary community has played an important role in facilitating our collaboration. Please join us in discovering more intriguing connections!

ACKNOWLEDGMENTS. Grigoriy Blekherman was partially supported by the National Science Foundation (NSF) grant DMS-1901950, Gregory G. Smith was partially supported by the Natural Sciences and Engineering Research Council of Canada (NSERC), and Mauricio Velasco was partially supported by proyecto INV2018-50-1392 from Facultad de Ciencias, Universidad de los Andes.

\section{References}

[1] Emil Artin, Über die Zerlegung definiter Funktionen in Quadrate (German), Abh. Math. Sem. Univ. Hamburg 5 (1927), no. 1, 100-115, DOI 10.1007/BF02952513. MR3069468

[2] Grigoriy Blekherman, João Gouveia, and James Pfeiffer, Sums of squares on the hypercube, Math. Z. 284 (2016), no. 12, 41-54, DOI 10.1007/s00209-016-1644-7. MR3545483

[3] Grigoriy Blekherman, Sadik Iliman, Martina JuhnkeKubitzke, and Mauricio Velasco, Gap vectors of real projective varieties, Adv. Math. 283 (2015), 458-472, DOI 10.1016/j.aim.2015.07.015. MR3383808
[4] Grigoriy Blekherman, Pablo A. Parrilo, and Rekha R. Thomas (eds.), Semidefinite optimization and convex algebraic geometry, MOS-SIAM Series on Optimization, vol. 13, Society for Industrial and Applied Mathematics (SIAM), Philadelphia, PA; Mathematical Optimization Society, Philadelphia, PA, 2013. MR3075433

[5] Grigoriy Blekherman, Daniel Plaumann, Rainer Sinn, and Cynthia Vinzant, Low-rank sum-of-squares representations on varieties of minimal degree, Int. Math. Res. Not. IMRN 1 (2019), 33-54, DOI 10.1093/imrn/rnx113. MR4023754

[6] Grigoriy Blekherman, Rainer Sinn, and Mauricio Velasco, Do sums of squares dream of free resolutions?, SIAM J. Appl. Algebra Geom. 1 (2017), no. 1, 175-199, DOI 10.1137/16M1084560. MR3633773

[7] Grigoriy Blekherman, Gregory G. Smith, Rainer Sinn, and Mauricio Velasco, Sums of squares and quadratic persistence on real projective varieties, J. Eur. Math. Soc. (JEMS) (to appear).

[8] Grigoriy Blekherman, Gregory G. Smith, and Mauricio Velasco, Sums of squares and varieties of minimal degree, J. Amer. Math. Soc. 29 (2016), no. 3, 893-913, DOI 10.1090/jams/847. MR3486176

[9] Grigoriy Blekherman, Gregory G. Smith, and Mauricio Velasco, Sharp degree bounds for sum-of-squares certificates on projective curves (English, with English and French summaries), J. Math. Pures Appl. (9) 129 (2019), 61-86, DOI 10.1016/j.matpur.2018.12.010. MR3998790

[10] Samuel Burer and Renato D. C. Monteiro, A nonlinear programming algorithm for solving semidefinite programs via low-rank factorization: Computational semidefinite and second order cone programming: the state of the art, Math. Program. 95 (2003), no. 2, Ser. B, 329-357, DOI 10.1007/s10107002-0352-8. MR1976484

[11] Man Duen Choi, Tsit Yuen Lam, and Bruce Reznick, Real zeros of positive semidefinite forms. I, Math. Z. 171 (1980), no. 1, 1-26, DOI 10.1007/BF01215051. MR566480

[12] Lynn Chua, Daniel Plaumann, Rainer Sinn, and Cynthia Vinzant, Gram spectrahedra, Ordered algebraic structures and related topics, Contemp. Math., vol. 697, Amer. Math. Soc., Providence, RI, 2017, pp. 81-105, DOI 10.1090/conm/697/14047. MR3716067

[13] David Eisenbud and Joe Harris, On varieties of minimal degree (a centennial account), Algebraic geometry, Bowdoin, 1985 (Brunswick, Maine, 1985), Proc. Sympos. Pure Math., vol. 46, Amer. Math. Soc., Providence, RI, 1987, pp. 3-13, DOI $10.1090 /$ pspum/046.1/927946. MR927946

[14] Christoph Hanselka and Rainer Sinn, Positive semidefinite univariate matrix polynomials, Math. Z. 292 (2019), no. 1-2, 83-101, DOI 10.1007/s00209-018-2137-7. MR3968894

[15] David Hilbert, Ueber die Darstellung definiter Formen als Summe von Formenquadraten (German), Math. Ann. 32 (1888), no. 3, 342-350, DOI 10.1007/BF01443605. MR1510517

[16] David Hilbert, Über ternäre definite Formen (German), Acta Math. 17 (1893), no. 1, 169-197, DOI 10.1007/BF02391990. MR1554835

[17] David Hilbert, Hermann Minkowski, Math. Ann. 68 (1910), no. 4, 445-471. MR1511572 
[18] Anthony Iarrobino and Vassil Kanev, Power sums, Gorenstein algebras, and determinantal loci, Lecture Notes in Mathematics, vol. 1721, Springer-Verlag, Berlin, 1999. Appendix C by Iarrobino and Steven L. Kleiman. MR1735271

[19] Monique Laurent and Antonios Varvitsiotis, A new graph parameter related to bounded rank positive semidefinite matrix completions, Math. Program. 145 (2014), no. 1-2, Ser. A, 291-325, DOI 10.1007/s10107-013-0648-x. MR3207690

[20] Henri Lombardi, Daniel Perrucci, and Marie-Françoise Roy, An elementary recursive bound for effective Positivstellensatz and Hilbert's 17th problem, Mem. Amer. Math. Soc. 263 (2020), no. 1277, v+125, DOI 10.1090/memo/1277. MR4071235

[21] Pablo A. Parrilo and Rekha R. Thomas (eds.), Sum of squares: theory and applications. AMS short course, Baltimore, MD, USA, January 14-15, 2019, Proceedings of Symposia in Applied Mathematics, vol. 77, Amer. Math. Soc., Providence, RI, 2020.

[22] Albrecht Pfister, Zur Darstellung definiter Funktionen als Summe von Quadraten (German), Invent. Math. 4 (1967), 229-237, DOI 10.1007/BF01425382. MR222043

[23] Bruce Reznick, Some concrete aspects of Hilbert's 17th Problem, Real algebraic geometry and ordered structures (Baton Rouge, LA, 1996), Contemp. Math., vol. 253, Amer. Math. Soc., Providence, RI, 2000, pp. 251-272, DOI 10.1090/conm/253/03936, MR1747589

[24] Claus Scheiderer, Sum of squares length of real forms, Math. Z. 286 (2017), no. 1-2, 559-570, DOI 10.1007/s00209016-1773-Z MR3648509

[25] Konrad Schmüdgen, The moment problem, Graduate Texts in Mathematics, vol. 277, Springer, Cham, 2017. MR3729411

[26] Lieven Vandenberghe and Martin S. Andersen, Chordal graphs and semidefinite optimization, Found. Trends Optim. 1 (2015may), no. 4, 241-433.

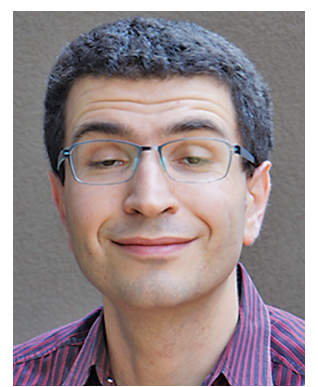

Grigoriy

Blekherman

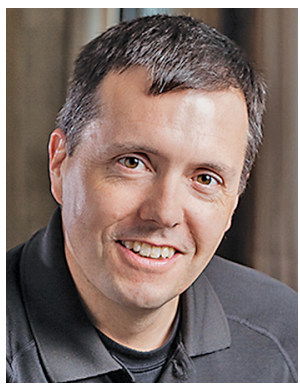

Gregory G. Smith

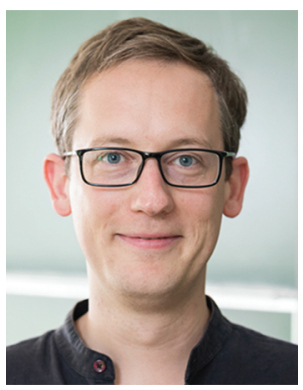

Rainer Sinn

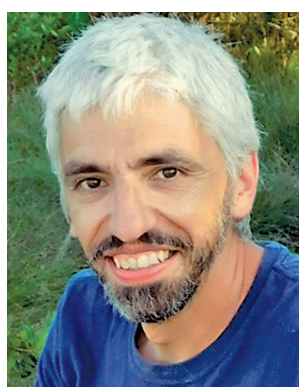

Mauricio Velasco

Credits

All figures and the opening image are courtesy of the authors. Photo of Grigoriy Blekherman is courtesy of Sung Ha Kang. Photo of Rainer Sinn is courtesy of Kay Herschelmann.

Photo of Gregory G. Smith is courtesy of Greg Black/Department of Mathematics and Statistics at Queen's University. Photo of Mauricio Velasco is courtesy of Florencia Reali. 